\title{
Haptic Device System For Upper Limb Motor Impairment Patients: Developing And Handling In Healthy Subjects
}

\author{
Tasuku Miyoshi ${ }^{1}$, Yoshiyuki Takahashi² ${ }^{2}$ Hokyoo Lee ${ }^{2}$, Takafumi Terada ${ }^{3}$, \\ Yuko Ito $^{4}$, Kaoru Inoue ${ }^{4} \&$ Takashi Komeda ${ }^{1}$ \\ 1. Shibaura Institute of Technology, JAPAN \\ 2. Toyo University, JAPAN \\ 3. Mitsubishi Precision Co. Ltd., JAPAN \\ 4. Tokyo Metropolitan University of Health Sciences, JAPAN
}

\section{Introduction}

Based on an aging society with fewer children, an explosive increase in the number of older adults and patients with motor dysfunctions and a reduction in the number of caregivers are anticipated. Patients with motor dysfunctions can benefit from movement therapies. They may in some cases gain social independence and improvements in daily living and quality of life. When a stroke damages the motor pathways for the arm and leg, the prospects for functional recovery are better for the leg than for the arm. It has been reported that 25 of 33 individuals with spinal cord injuries learned to walk independently within 3 to 20 weeks (median 10.5) with partial body weight support treadmill training, known as Lauband therapy

$\varepsilon$ (Wernig \& Muller, 1992). However, this treatment presents specific challenges for the therapist.

0 The passive moving of a disabled person's legs is ergonomically difficult since the individual $\Phi$ being treated cannot independently support and/or move his legs. In order to improve this 铕 therapy, a driven gait orthosis was developed for use on patients with varying degrees of o paresis or spasticity for up to one-half hour (Colombo et al., 2000). For the sake of basic human O movements, e.g., standing upright with stability or maintaining a steady gait, lower limb r. rehabilitation is an important aspect of regaining function.

From the viewpoint of assistance with daily activities, however, it is obvious that upper limb rehabilitation is also important, especially for stroke patients. There are several \& rehabilitation protocols: increased intensity of treatment (Lincoln et al., 1999), of device-enhanced treatment (Sunderland et al., 1994; Feys et al., 1998), and specially focused \% sensorimotor activity for the affected limb, known as "constraint-induced movement Tू therapy" (Miltner et al., 1999). In the case of constraint-induced movement therapy, a clinical of trial with acute rehabilitation is feasible and is associated with less arm impairment at the \& end of treatment (Dromerick et al., 2000; Taub et al., 1993). In this respect, increased \& sensorimotor activity involving a paralyzed upper limb after a stroke leads to improved motor performance and enhanced activities of daily living.

$\underset{\mathcal{D}}{\widetilde{D}}$ Several groups are approaching upper limb rehabilitation with the use of robotics and information technology to control sensorimotor activity delivered to an upper limb 
paralyzed after a stroke. For example, MIT-MANUS (Aisen et al., 1997; Krebs et al., 2004) and Mirror Image Movement Enabler (Lum et al., 2006) are now well documented. To our knowledge, however, it is still unclear whether those kinds of applications are more effective for robotic rehabilitation than traditional methods. In addition, the effects of the assist/resist forces as subjects execute exercise protocols are still unknown, whereas robotic rehabilitation has some advantages in easily changing the intensity of rehabilitation exercises. To solve these problems, we designed a haptic device by applying virtual-reality technology as a robotic rehabilitation apparatus.

In this paper, we show our newly designed haptic device and some of its applications. We then demonstrate our preliminary experimental results achieved by healthy young subjects. Our goal was to apply the haptic device as a neurorehabilitation method with a reduction in the work load of the therapist. Therefore, in the last part of this paper, we describe the clinical relevance of using the haptic device in robotic rehabilitation. Parts of this research have been reported elsewhere (Takahashi et al., 2003; Lee et al., 2005).

\section{Haptic device for upper limb motor rehabilitation}

\subsection{Concept behind the haptic device}

We tried to develop a functional motor rehabilitation system using robotics and information technology. The haptic device was designed on the assumption that it is mainly used by patients alone or under the supervision of a therapist. In order to help the patients, this system is designed so that either the therapist or the patient can set up the personal data.

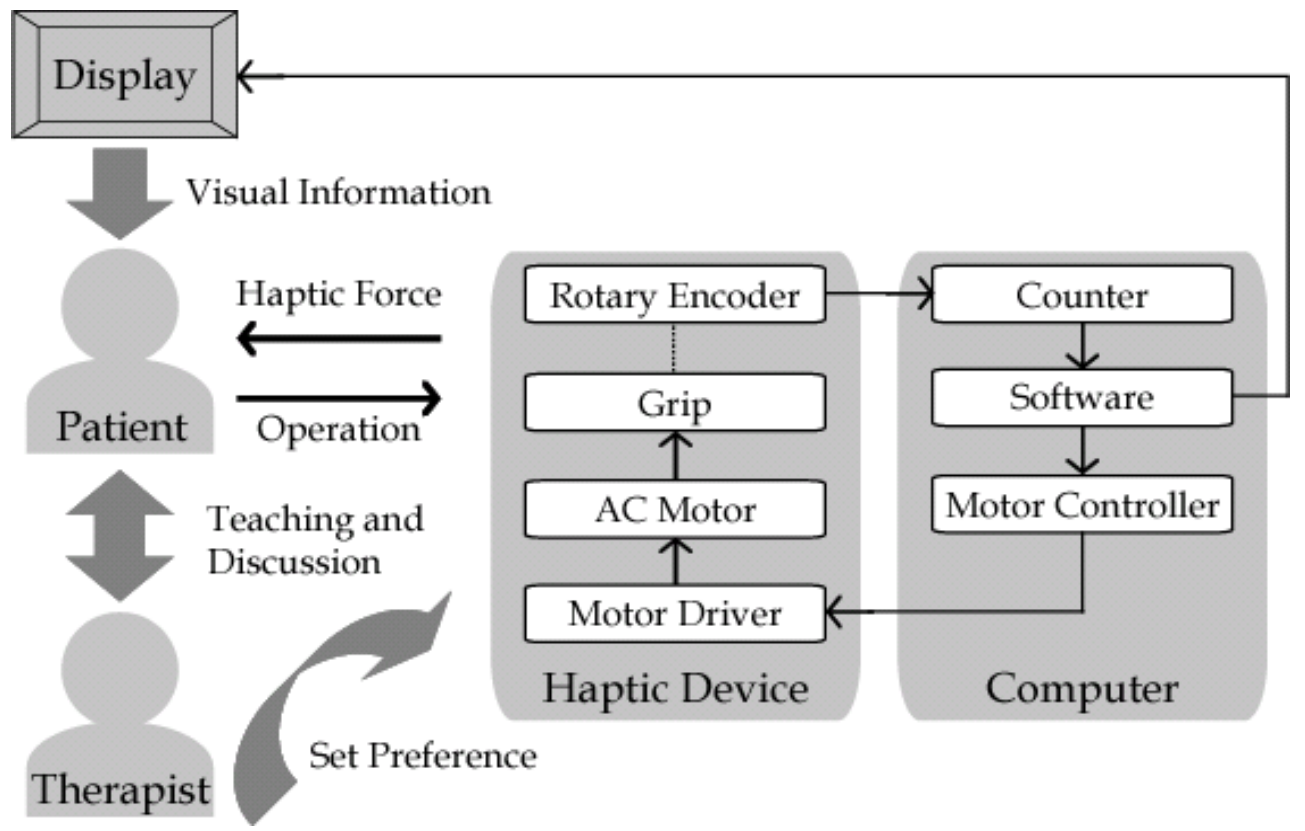

Fig. 1. Schematic relationships among the patient, the therapist, and the haptic device. 
It is necessary for clinicians or therapists to make decisions with regard to safety and effective training, where the enhanced motor improvement results in the patients losing their concentration. Hence, a robotic rehabilitation system including a computer would record the training data and evaluate the level of recovery with regard to a motor dysfunction for further qualitative analysis.

The schematic relationships among patients, therapists, and the haptic device are shown in Fig. 1. Either the patients or the therapists select the training program shown on the monitor. The display shows both the selected program and the current status, while the patient conducts the training. After training, the patients and therapists discuss the outcome with regard to the day's activity and the progression of improvement. In addition, future directions and/or self-contemplation using the haptic device is discussed at this stage.

\subsection{Design of the Haptic device}

The haptic device consists of two servomotors with reduction gears and optical encoders, four link rods, a flat panel, and two man-machine interfaces; one is the monitor display, and the other one is the grip. Figure 2 is an overview of the haptic device.

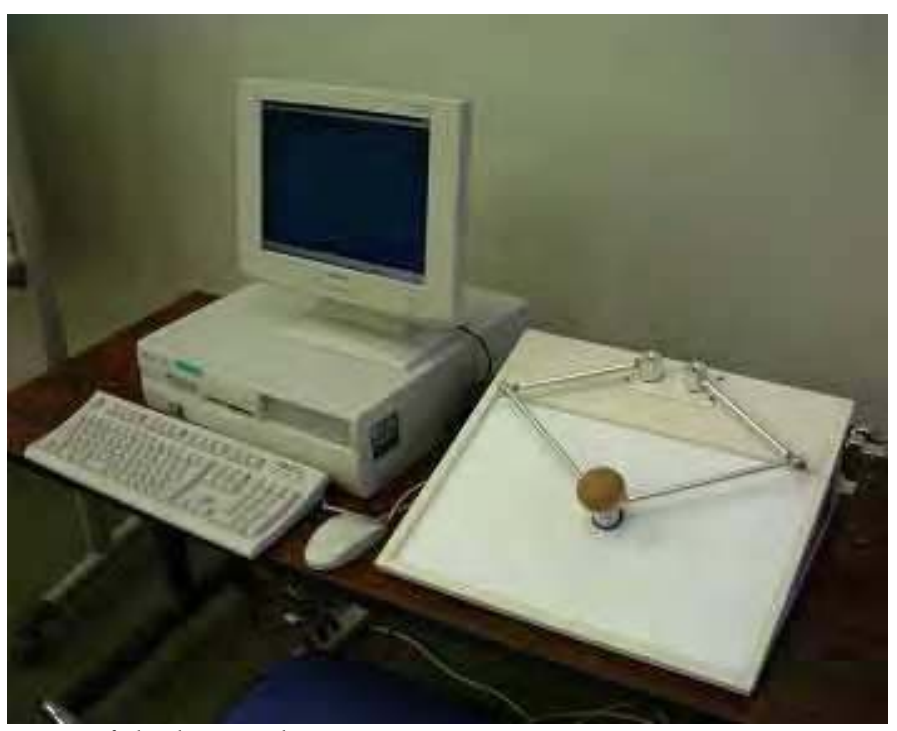

Fig. 2. An overview of the haptic device system.

The size of the haptic device is $430 \mathrm{~mm}$ in depth, $500 \mathrm{~mm}$ in width, and $190 \mathrm{~mm}$ in height. The moving range is $400 \mathrm{~mm}$ in the medio-lateral direction and $250 \mathrm{~mm}$ in the anterior-posterior direction. The length of the link rods is $190 \mathrm{~mm}$ for the motor side and 230 $\mathrm{mm}$ for the grip side. The grip and servomotors are connected by the link rods. Patients can move the grip on the surface of the flat panel and train their upper-limb movements in the planar horizontal plane. If the patient moves the grip of the haptic device, the cursor on the display moves simultaneously with the grip, and the patient can feel the virtual force applied to the grip. The current position of the grip can be calculated by the encoder pulse count and the length of the link rods. Figure 3 is a schematic figure of the haptic device used to calculate the current position of the grip $(x, y)$ in a planar horizontal plane as follows, 


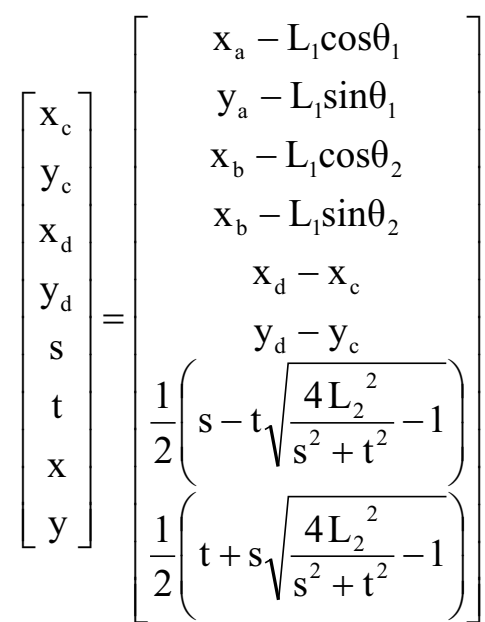

where all of the symbols are followed in Fig. 3. The theoretical resolution of the grip position is $0.01 \mathrm{~mm}$. The haptic device can deliver a maximum force of $30 \mathrm{~N}$ onto the hand grip in each direction. The 15-inch LCD is used for displaying the training program and the current position of the cursor. The aspect ratio of the display work field is in proportion to the actual flat panel.

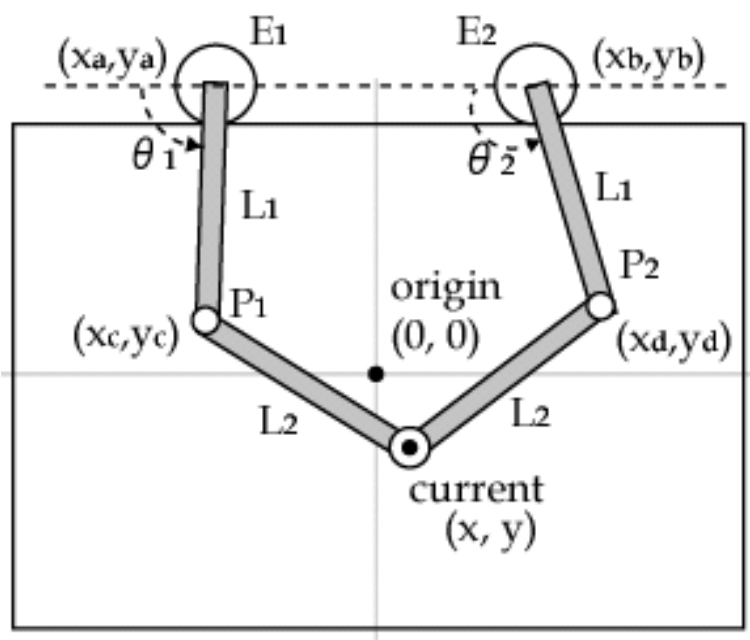

Fig. 3. A schematic figure of the haptic device in a planar horizontal plane. E1 and E2 denote the position of the axis of the servomotor; L1=190 mm; L2=230 mm; P1 and P2 denote the joint point between two link rods.

The haptic device provides six types of haptic force, as follows: load (Fl), assistance (Fa), spring (Fs), viscosity (Fv), friction (Ff), and special-effect force (Fe). The operator (usually the therapist) can change the types of haptic forces and their magnitudes according to the disorder levels of the users. The load/assistance forces were generated in the opposite/same direction of the grip velocity vector. The magnitudes of these forces 
increased in proportion to the distance between the current grip position and the target position. The load force $\mathrm{Fl}$ and the assistance force $\mathrm{Fa}$ are given as follows,

$$
\mathrm{F}_{1}=\left[\begin{array}{c}
\mathrm{F}_{\mathrm{lx}} \\
\mathrm{F}_{\mathrm{ly}}
\end{array}\right]=\mathrm{K}_{1}\left(\left[\begin{array}{l}
\mathrm{x}_{0} \\
\mathrm{y}_{0}
\end{array}\right]-\left[\begin{array}{l}
\mathrm{x} \\
\mathrm{y}
\end{array}\right]\right)
$$

where the current grip position is $(x, y)$, the target position is $(x 0, y 0)$, and the gain is $\mathrm{Kl}$. In the case of the assistance force, force Fa and gain $\mathrm{Ka}$ are applied instead of Fl and Kl. The spring force is generated in the direction of the initial grip position. The magnitude of this force increases in proportion to the distance between the current position and initial position of the grip. Spring force Fs is the same as (1), where gain $\mathrm{Kl}$ is replaced by Ks. The viscosity and the friction force are generated in the opposite direction of the grip velocity vector. The magnitude of the viscosity force increases in proportion to the velocity of the grip, whereas that of the friction force is constant. The viscosity force Fv and the friction force Ff are given as follows,

$$
\begin{gathered}
F_{v}=\left[\begin{array}{c}
F_{v x} \\
F_{v y}
\end{array}\right]=K_{v}\left[\begin{array}{c}
\dot{x} \\
\dot{y}
\end{array}\right] \\
F_{f}=\left[\begin{array}{c}
F_{f x} \\
F_{f y}
\end{array}\right]=K_{f}\left[\begin{array}{c}
\frac{\dot{x}}{|\dot{x}|} \\
\frac{\dot{y}}{|\dot{y}|}
\end{array}\right]
\end{gathered}
$$

where the velocity of the grip is and the gain is $\mathrm{Kv}$ for the viscosity and $\mathrm{Kf}$ for the friction force. In order to provide special effects in some training programs, e.g., the contact force of the wall or the reaction force when hitting some objects, this special-effect force Fe was generated. The total haptic force on grip $F$ was the summation of each force, as follows,

$$
\mathrm{F}=\mathrm{F}_{1}+\mathrm{F}_{\mathrm{a}}+\mathrm{F}_{\mathrm{s}}+\mathrm{F}_{\mathrm{v}}+\mathrm{F}_{\mathrm{f}}+\mathrm{F}_{\mathrm{e}}
$$

\subsection{Implemented training programs}

The haptic device is controlled with a PC/AT computer and implemented control software. The control software achieves the following functions: controlling the haptic device, displaying the training program and the current position of the cursor on the monitor, acquiring the training data for each patient, and evaluating the training results. Of course, the cursor on the display moves in synchronicity with the grip of the haptic device, and the system provides a haptic force that obeys Eq. (5). The data acquisition program stores the training data, such as the time and grip position. The stored data could be used for offline analysis in further quantitative assessment of the functional motor rehabilitation, since the evaluation program is now under construction.

The implemented training now available consists of five different basic training programs and two applied training programs. In the upper left column of Fig. 4, the point-to-point movement training program is shown. In this training, nine circles are shown on the display, and patients try to move the cursor from circle to circle. In the upper middle column of Fig. 4 , the line drawing training program is shown. Nine small circles and lines that connect the circles are shown on the display, and patients try to move the cursor from circle to circle while staying on the lines.

In the upper right column in Fig. 4, the circle-drawing training program is shown. Four concentric doughnut rings are shown on the display, and patients try to move the cursor 
while staying on each doughnut ring. In the bottom left column in Fig. 4, the wave-drawing training program is shown. Two small circles and a sinuous wavy line are shown on the display, and the circles are connected with the wavy line.

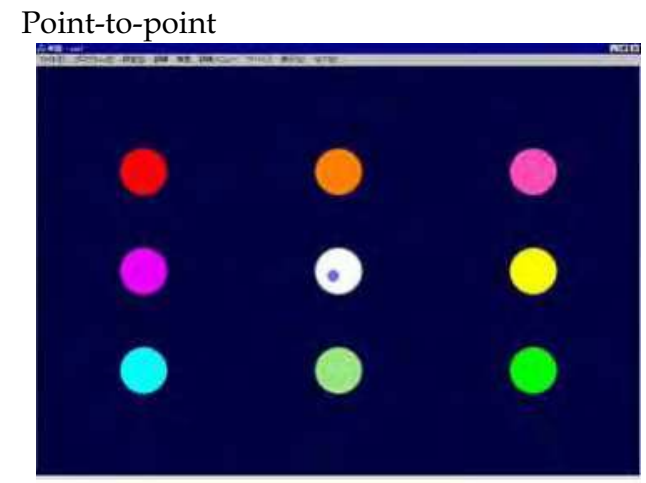

Line

Circle drawing

Wave shape drawing
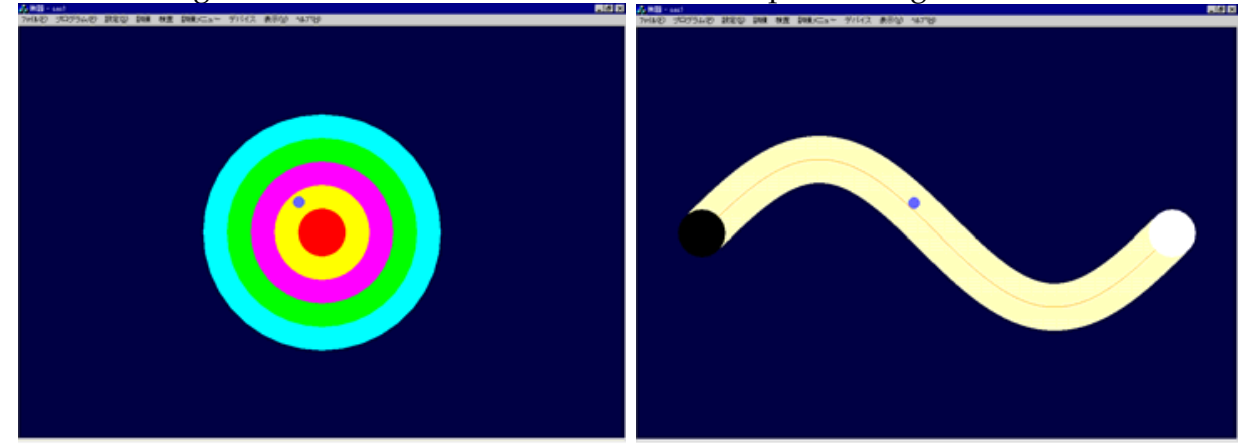

Tile moving

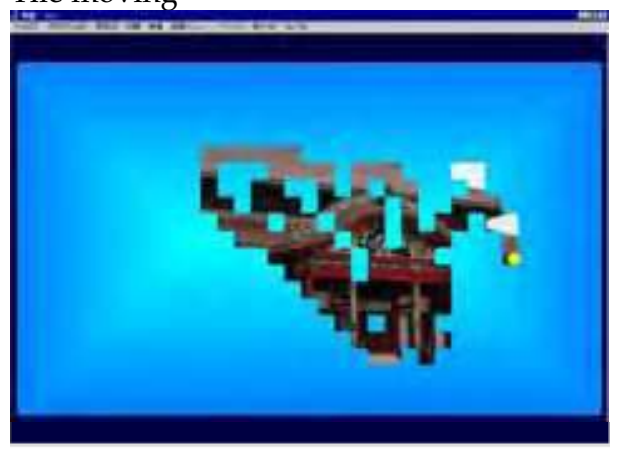

Fig. 4. Basic training programs displayed on the monitor.

Patients try to move the cursor from one circle to the other while staying on the line. In the bottom right column of Fig. 4, the tile-moving training program is shown. Square colored tiles are shown on the display, and patients try to move the cursor over all tiles. When the 
cursor is over a tile, the color of the tile becomes clear, and a hidden picture appears. The displacement and the radius of the circles, the radius and the width of the doughnut rings, the amplitude and the cycle of the waves, the number of tiles, the magnitude and the direction of the virtual force, and the hidden picture are all changeable by the operators. While the patients execute the basic training programs, some patients might become bored and lose their concentration on the training program. Therefore, we constructed further applied training programs involving hockey and maze games. These may help the patients not lose their motivation and maintain their concentration while training. On the left in Fig. 5, a hockey game is displayed on the monitor. The hockey game is similar to a table hockey game, and patients try to hit a puck and score against the computer program. Various haptic forces are delivered when moving the grip, e.g., impact, viscous, and spring forces. On the right in Fig. 5, a maze game is shown. Patients try to move the cursor from start to goal by going through a complex route. When the cursor hits the wall of the maze, patients feel the haptic forces and are not able to get over the wall. The velocity of the puck and the intensity and direction of the applied force are also changeable by the operators. These programs are effective for functional cognitive rehabilitation.
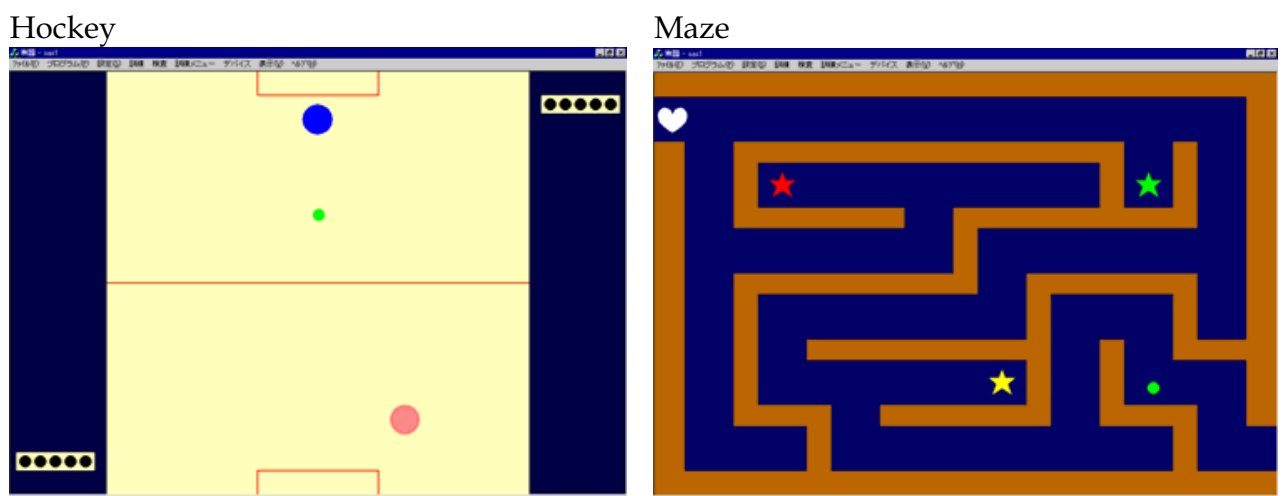

Fig. 5. Applied training programs displayed on the monitor.

\section{Handling the haptic device in healthy subjects}

\subsection{Objective of the preliminary experiment}

As reported in the Introduction, the effects of the assist/resist forces as the subjects execute their motor rehabilitation in a planar horizontal plane are still unknown. The virtual force relationships may play an important role in motor rehabilitation when making decisions on safe and effective robotic rehabilitation. Hence, the purpose of this preliminary experiment was to investigate the relationships between the assisting and the resisting force acting on the grip while subjects moved their grip from one point to another.

\subsection{Methods}

Nine healthy right-handed males (21 to 24 years of age) participated in this preliminary experiment. They had no known history of neurological disorders and gave informed consent approved by the local Ethics Committee of Shibaura Institute of Technology. Each subject was seated comfortably on a height-adjustable chair with his trunk immobilized by a strap and the wrist of his right forearm attached to the forearm support base with Velcro straps so that the 
elbow and shoulder rotation were in a horizontal plane. When the cursor displayed on the monitor was at the start position, the elbow joint angle was at $110 \mathrm{deg}$ flexion $(0 \mathrm{deg}$ : full elbow extension), the shoulder angle was at $60 \mathrm{deg}$ abduction and $35 \mathrm{deg}$ medial rotation (0 deg: fundamental position), and the wrist and shoulder joints lined up straight in a horizontal plane. The subjects were then instructed to move the cursor from the start point to the mid-point (forward-motion phase) and from the mid-point to the goal position (backward-motion phase) along with the target line ( $30 \mathrm{~mm}$ width shown on the monitor) in a horizontal plane. The distance from the start to the mid-point was $200 \mathrm{~mm}$; thus, the total travel distance was $400 \mathrm{~mm}$ in one cycle. In this preliminary experiment, the applied force was set at $2.5 \mathrm{~N}$ and was given constantly from the desired direction to the grip. Three force conditions (no-assist, anterior-to-posterior, and posterior-to-anterior; duration, $30 \mathrm{sec}$ in each) were executed at random. On account of this, the anterior-to-posterior force condition became an assist force in the forward-motion phase and a resist force in the backward-motion phase. In fact, it showed opposite aspects in the posterior-to-anterior force condition. Figure 6 is a schematic figure for these preliminary experimental conditions with the direction of the applied force in light-gray arrows. These training sets were conducted 5 minutes per day and repeated for 5 days. All of the subjects carried out point-to-point movements at a self-determined comfortable speed each day. The number of the cycles per day varied day by day because the performance speeds tended to increase as training progressed.
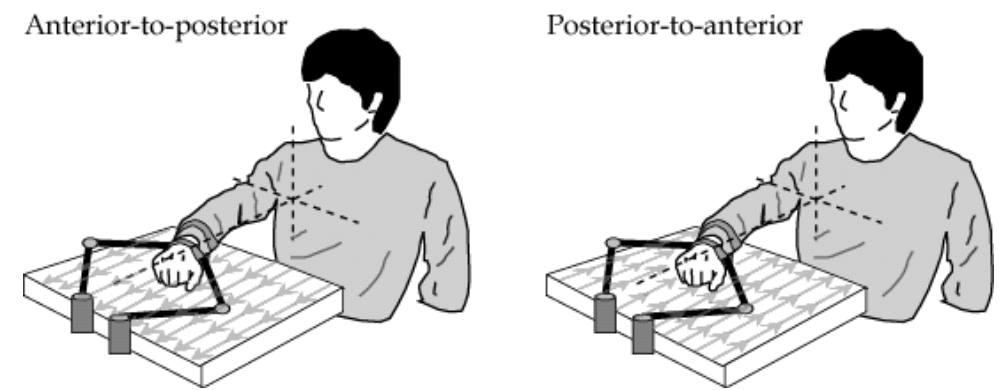

Fig. 6. Schematic figure for the experiment; (left) for the anterior-to-posterior applied force condition and (right) for the posterior-to-anterior applied force condition. The light-gray arrows denote the direction of the applied force.

On the first and last days, the surface EMG activities were recorded from the bellies of the biceps brachii (BB) and the triceps brachii (TB) muscles using pairs of $\mathrm{Ag}-\mathrm{AgCl}$ electrodes (7 $\mathrm{mm}$ diameter; $3 \mathrm{~cm}$ inter-electrode distance). The EMG signals were amplified and band-pass-filtered $(30 \mathrm{~Hz}-5 \mathrm{kHz})$ with a bioelectric amplifier (AB-610J Nihon Kohden Co., Ltd.). All the data were stored in a personal computer at a sampling frequency of $100 \mathrm{~Hz}$ for both the current position $(x, y)$ and the grip forces and $1 \mathrm{kHz}$ for the EMG signals for off-line analysis. The EMG signals were then full-wave-rectified after subtracting the DC component. Both the forward- and backward-motion phases were expressed in relation to the $100 \%$ in each phase. The mean velocities and the positional errors, which were expressed as regression errors, for the forward- and the backward-motion phases were calculated from the cursor position data. Table 1 contains the details of the direction of the elbow and the shoulder joint movement and the expected EMG changes under the anterior-to-posterior/posterior-to-anterior applied force condition during the forward-/backward-motion phase. 
Haptic Device System For Upper Limb Motor Impairment Patients: Developing And Handling In Healthy Subjects

\begin{tabular}{|c|c|c|c|c|c|c|c|}
\hline & \multicolumn{2}{|c|}{ Elbow } & Shoulder & \multicolumn{2}{|c|}{ Anterior-to-posterior } & \multicolumn{2}{|c|}{ Posterior-to-anterior } \\
\hline \multirow{2}{*}{ Forward } & \multirow{2}{*}{ Extension } & Agonist : TB & \multirow{2}{*}{ Inner rotation } & \multirow{2}{*}{ Assist } & BB : no change & \multirow{2}{*}{ Resist } & BB : no change \\
\hline & & Antagonist : BB & & & TB : decrease & & TB : increase \\
\hline \multirow{2}{*}{ Backward } & \multirow{2}{*}{ Flexion } & Agonist : BB & \multirow{2}{*}{ Extorsion } & \multirow{2}{*}{ Resist } & BB : increase & \multirow{2}{*}{ Assist } & BB : decrease \\
\hline & & Antagonist : TB & & & TB : no change & & TB : no change \\
\hline
\end{tabular}

Table 1. Direction of the elbow and the shoulder joint angular displacement movements and expected EMG changes in anterior-to-posterior/posterior -to-anterior applied force condition during the forward-/backward-motion phase.

To investigate the effects of either the assist or the resist force to the EMG activities, the mean amplitudes of both the BB and the TB EMG activities under the no-assist condition in each phase were used to normalize the amplitudes under the two other conditions. EMG coordination between bi-articular muscles was indispensable for a smooth pedaling performance (Raasch and Zajac, 1999). In this respect, it was considered for the case of the upper limb movements that the smooth two-joint movement in a planar horizontal plane was achieved by the EMG coordination within the BB and the TB. In this paper, therefore, we defined the area under the subtracted two time-series of the BB and the TB EMG activities as EMG coordination in the forward-/backward-motion phase, as follows,

$$
(\text { EMGCoordination })=\left|\frac{\mathrm{BB}(\mathrm{i}, \mathrm{t})}{\mathrm{RMS} \_\mathrm{BB}}-\frac{\mathrm{TB}(\mathrm{i}, \mathrm{t})}{\mathrm{RMS} \_\mathrm{TB}}\right|
$$

where BB(i,t) and TB(i,t) are the rectified EMG data in each phase, RMS_BB and RMS_TB are the root mean-squared EMG activities for the $\mathrm{BB}$ or the TB under the no-assist condition, and i denotes the forward- or the backward-motion phase. We then constructed a three-dimensional (3D) plot of the EMG coordination as a function of the mean velocity and the positional error. The EMG coordination can be expressed as a single plane on the 3D plot. When the positional errors increase and, as a result, EMG coordination decreases, the smoothness of the point-to-point movement is lost. In addition, the mean velocity and the positional error obey in a speed-accuracy tradeoff, also known as Fitt's law (Fitts, 1954). It will be demonstrated, however, that the EMG coordination is enhanced through training when the mean velocity increases rather than when there is an increase in the regression error and the EMG coordination. Namely, two slopes of each single plane, which were calculated as standardized partial regression coefficients using multiple regression tests, were used to quantify the contributions of the positional error and the mean velocity to the EMG coordination. One-way repeated analysis of variance was used to determine the significant differences among the no-assist, anterior-to-posterior, and posterior-to-anterior conditions. Levels were considered statistically significant at $P<0.05$.

\subsection{Results}

Figures 7-9 show a typical time series of the cursor positions on the XY-table, the cursor velocities, the EMG activities of the $\mathrm{BB}$, and the $\mathrm{TB}$ under three different applied force conditions in one subject. It is clear that the movement velocity and the achievement periods were increased after training for the same duration. As for the movement velocities, their profiles became sharper than those before training, since both the acceleration and braking 
phases became steeper during both the forward- and backward-motion phases and showed a bell shape after training.

In the case of the levels of the EMG activities, the BB, which is the agonist muscle during the backward-motion phase, was enhanced under the anterior-to-posterior applied force condition. In addition, the TB EMG activities increased against the posterior-to-anterior applied force and decreased under the anterior-to-posterior applied force condition. These phenomena are shown more clearly in Figs. 10 and 11: when the subjects executed forward/backward motion, the EMG activities of the agonist muscles were enhanced against the resist force, whereas the levels of the antagonist muscles were decreased by the assist force compared with those under the no-assist condition.

The phasic EMG activities between the $\mathrm{BB}$ and the TB were shown more clearly after training, considering that the EMG coordination with the $\mathrm{BB}$ and the TB might correlate positively with the enhanced movement velocities. A three-dimensional (3D) plot of the relationship among the EMG coordination, positional error, and movement velocity in one subject (Fig. 12.) is shown in Fig. 7. In this case, the EMG coordination increased with the increase of the movement velocities, whereas the same tendencies for positional errors were not observed under all three conditions.

The contributions of the movement velocity and the positional errors to the EMG coordination are denoted by the two standardized partial regression coefficients of the single plane on the 3D plot (Fig. 13.). Under each condition, the EMG coordination was more dependent on the movement velocity than on the positional errors, since the standardized partial regression coefficient for the movement velocity was positive. In addition, the standardized partial regression coefficient under the posterior-to-anterior applied force condition was greater than that of either the no-assist or the anterior-to-posterior applied force condition. For the positional errors, the standardized partial regression coefficient was close to zero under the posterior-to-anterior applied force condition.

\subsection{Discussion}

The main objective of this preliminary experiment was to evaluate the efficacy of assist/resist applied force for upper limb muscle activities with a two-joint arm movement in the planar horizontal plane while using the haptic device. The extent to which our results can be generalized is questionable because they were based on data obtained from only healthy right-handed young subjects.

The muscle activation patterns responsible for initiating hand movements are processed in the central nervous system on the basis of a simple representation of movement orientation, without taking into account the specific dynamic effects of the motion (Karst and Hasan, 1991a, b). Recent studies, however, have demonstrated that the cortical motor activity is continuously modulated while performing drawing movements to accommodate changes in orientation and velocity of the arm end-effector trajectory (Schwartz and Moran, 1999; Scheidt et al., 2000). Our results demonstrated that the effects provided by an assist/resist force were different. In these respects, it was considered that the forward-/backward-motion phase against the resist force needs more positive recruitment of the central nervous system under the anterior-to-posterior and/or posterior-to-anterior applied force condition than under the no-assist condition and, as a result, EMG activities were enhanced. Simultaneously, the decreased EMG activities following the assist force suggested the negative recruitment of the central nervous system. 


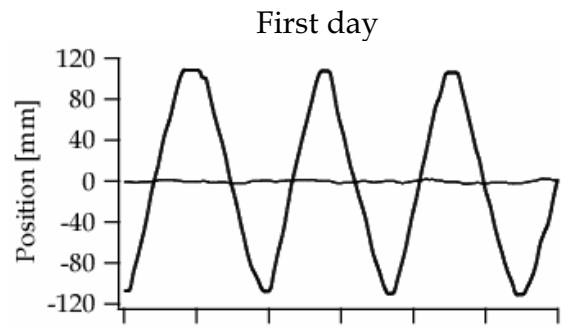

Last day
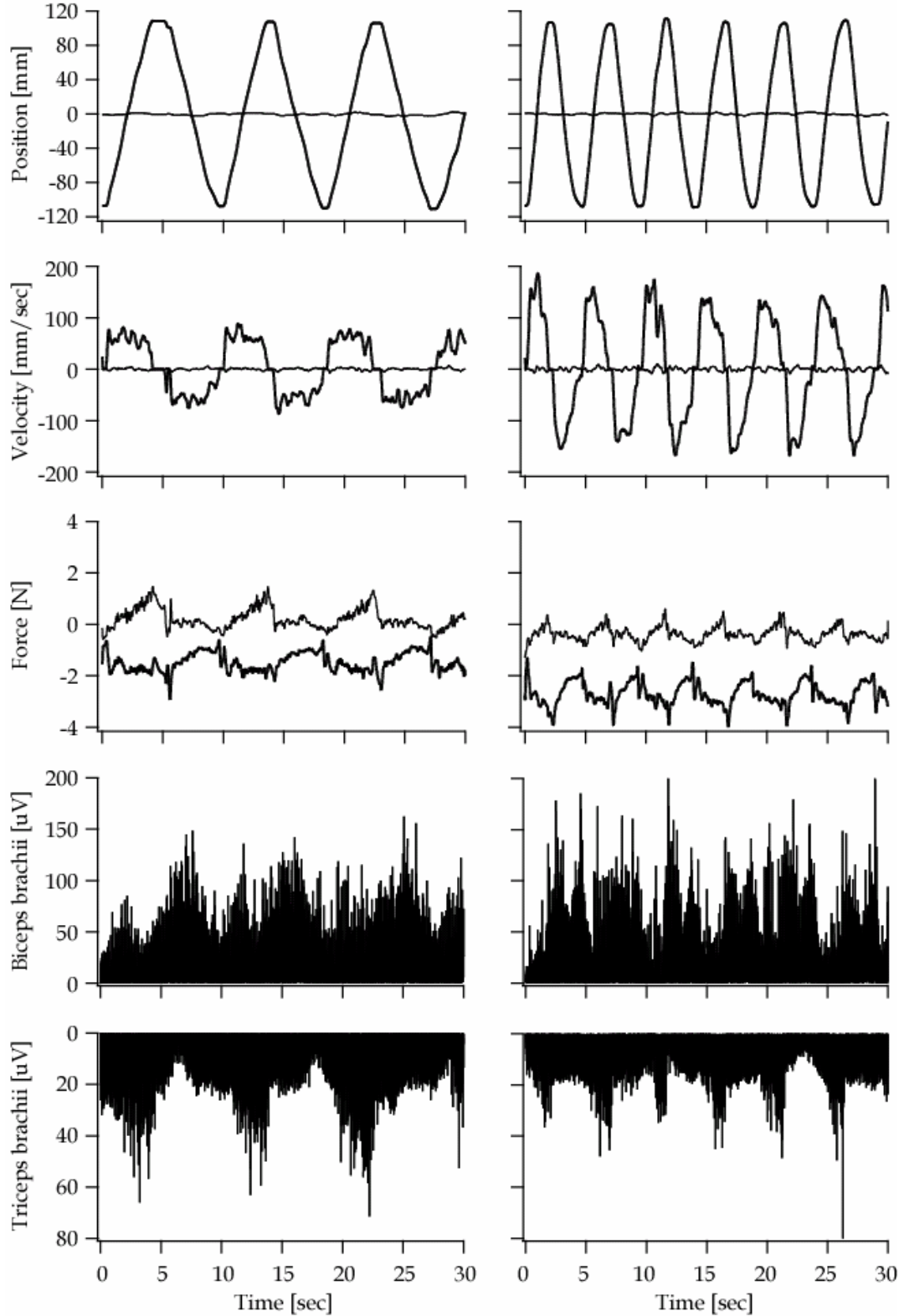

Fig. 7. The left panels show the typical time-series of the first-day waves under the no-assist condition; cursor positions (top column), velocities (middle upper column), force (middle column), and EMG activity of the BB (lower middle column) and the TB (bottom column) in one subject. The right panels show the last-day waves in the same subject. The thin lines denote $\mathrm{x}$-axial variables of position, velocity, and force, and the thick lines denote the $y$-axial variables. 

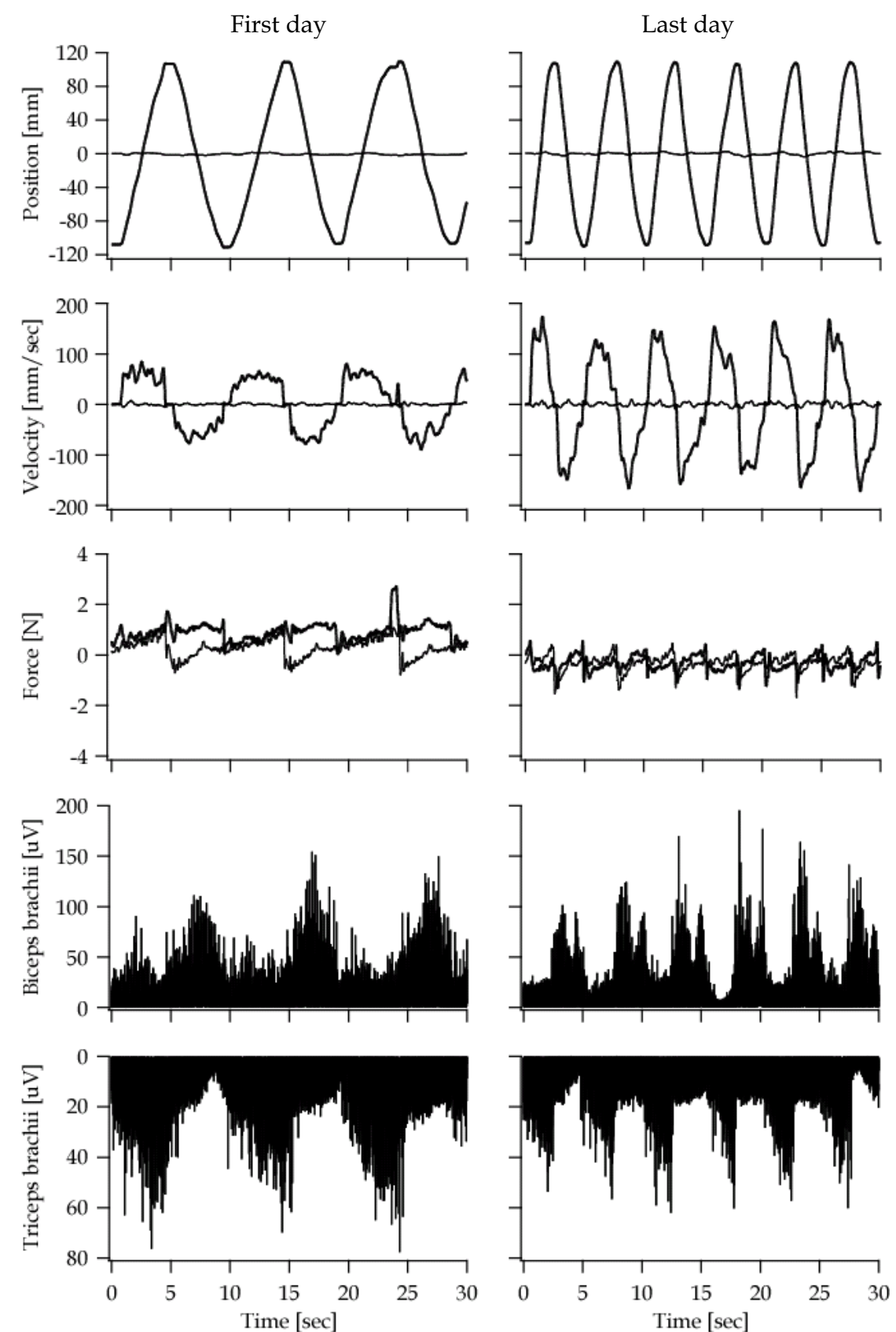

Fig. 8. Typical time-series under the anterior-to-posterior force applied condition in the same subject shown in Fig. 7. The orders of the panels and the lines are the same as those in Fig. 7. 


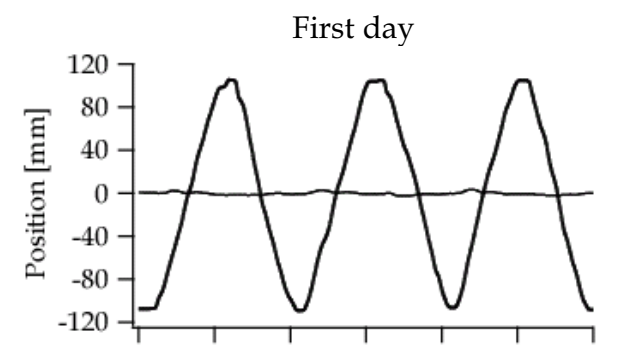

Last day
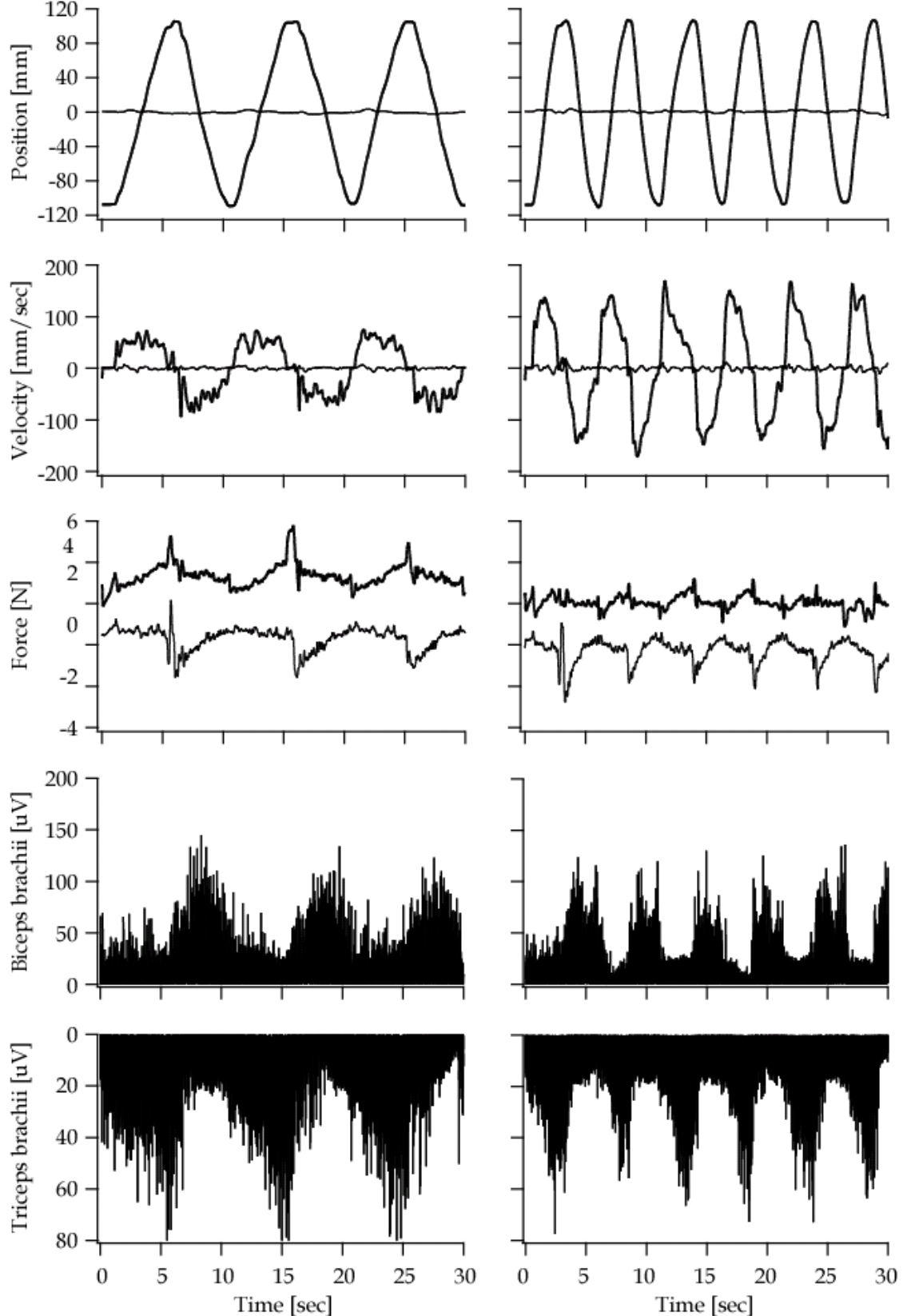

Fig. 9. Typical time-series under the posterior-to-anterior force applied condition in the same subject shown in Fig. 7. The orders of the panels and the lines are the same as those in Fig. 7. 

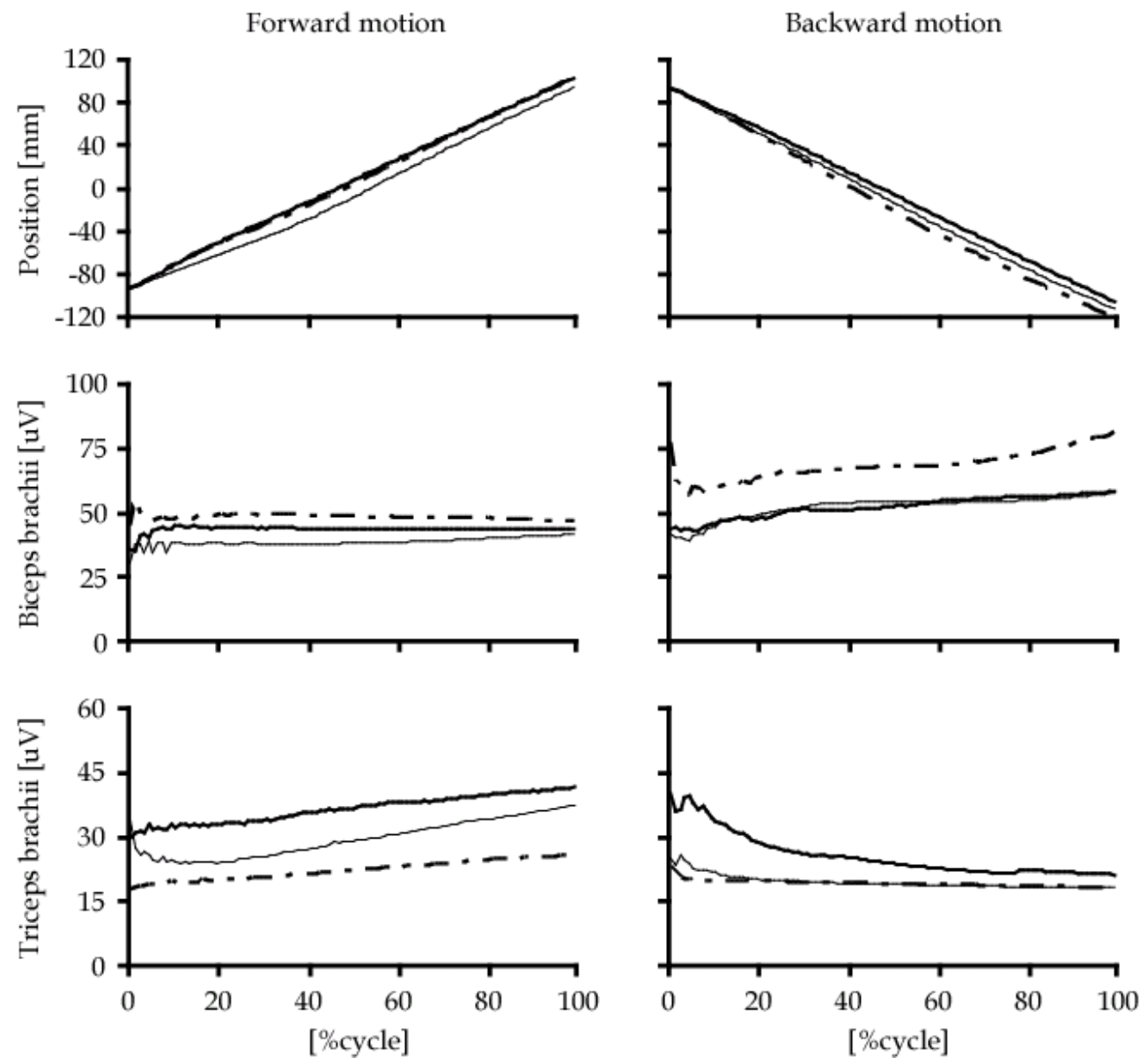

Fig. 10. The left panels show the ensemble-averaged waves in the forward-motion phases; cursor positions (top) and EMG activity of the BB (middle) and of the TB (bottom) in the same subject shown in Fig. 7. The right panels are those in the backward-motion phases. The thin lines denote the no-assist condition, the dashed lines show the anterior-to-posterior force applied condition, and the thick lines denote the posterior-to-anterior force applied condition.

In this study, we defined the EMG coordination as an index of the phasic relationships between agonist and antagonist muscles during the forward-/backward-motion phase. Thus, if the EMG coordination shows a large value, the effect to be provided by the agonist muscles is high against the demands of the movement task. The results suggest that the improvement in the movement speed after training was caused by the enhanced EMG coordination, since the contribution of the movement speed to the EMG coordination was significantly higher than that of the positional error. 
Forward motion
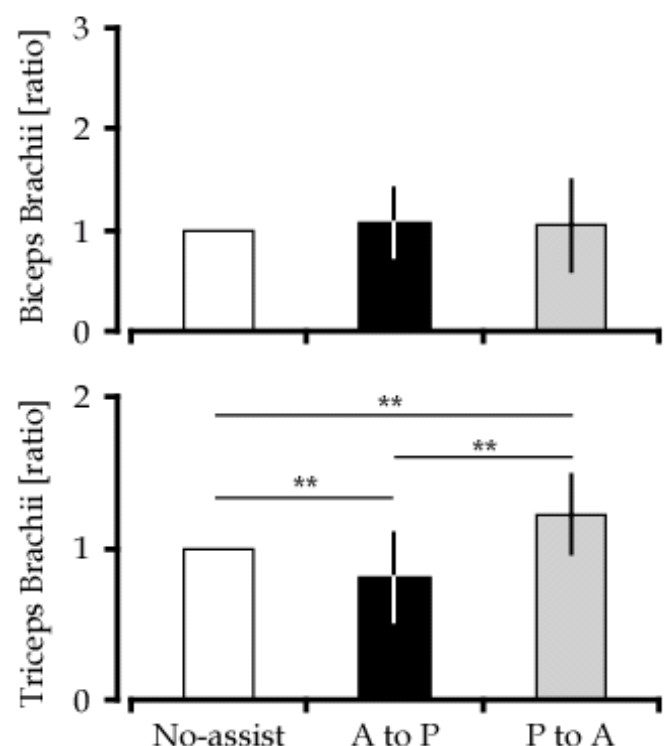

Backward motion
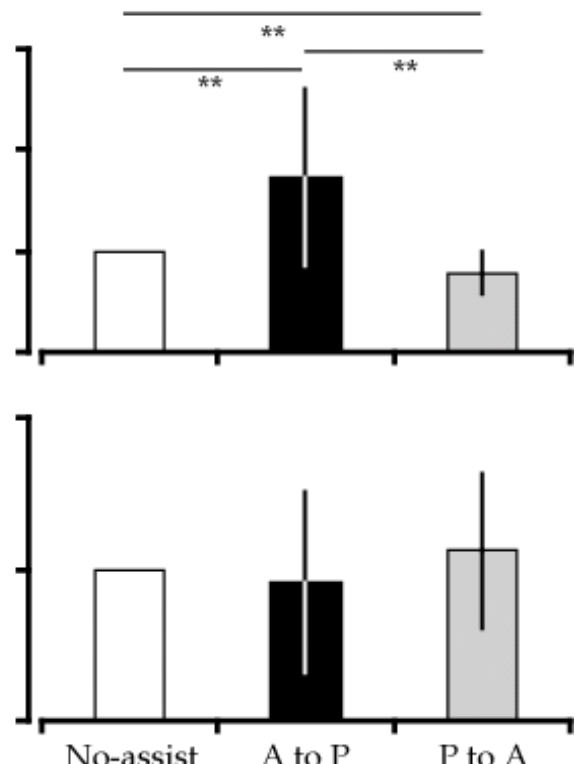

Fig. 11. Ratios of EMG activities of the BB and the TB under both the anterior-to-posterior and the posterior-to-anterior force applied conditions relative to the no-assist condition during the forward-motion phase (left) and the backward-motion phase (right). "A to $\mathrm{P}^{\prime \prime}$

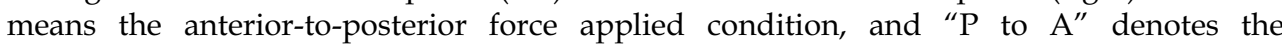
posterior-to-anterior force applied condition. (** $: P<0.01)$.

\section{Clinical relevance}

Although the haptic device provided only 2.5 N force, the EMG activities increased or decreased depending on the adjustment and the direction of the force. These phenomena suggested that the application of only $2.5 \mathrm{~N}$ would have a training effect on the muscle force and the motor planning about trajectories of the wrist position. EMG data provided evidence of improved muscle activation patterns between agonist and antagonist muscles by the application of an assist/resist force as well as of improved EMG coordination. EMG coordination between bi-articular muscles was indispensable for a smooth pedaling performance (Raasch and Zajac, 1999). Therefore, the haptic device could improve upper limb EMG coordination, especially, for bi-articular muscles, such as the BB and the TB.

In general, rehabilitation methods for stroke patients, e.g., peg boards and sanding boards, are implemented by therapists. Although these methods keep the therapists' workload at a low level, it might be difficult to keep the patients' motivation and/or concentration until rehabilitation is achieved. Through the use of the haptic device, training programs could be transformed into computer games or other games that would attract the users and maintain their motivation. These programs might be effective for functional cognitive rehabilitation. 


\section{Conclusion}

One critical question that should always be asked when considering rehabilitation methods is their duration, in other words, how long the training should last and/or whether the gains will persist for a significant period after training. In this respect, further studies are needed on the use of the haptic device as an upper limb rehabilitation method for stroke patients. EMG coordination would be an effective training index. Higher EMG coordination means the improvement of the upper limb movement at the neural control level, including the motor planning of the wrist position. Therefore, assessment of the improvement in the EMG coordination using the haptic device would indicate the length of effective training periods for the upper limb motor rehabilitation of stroke patients.
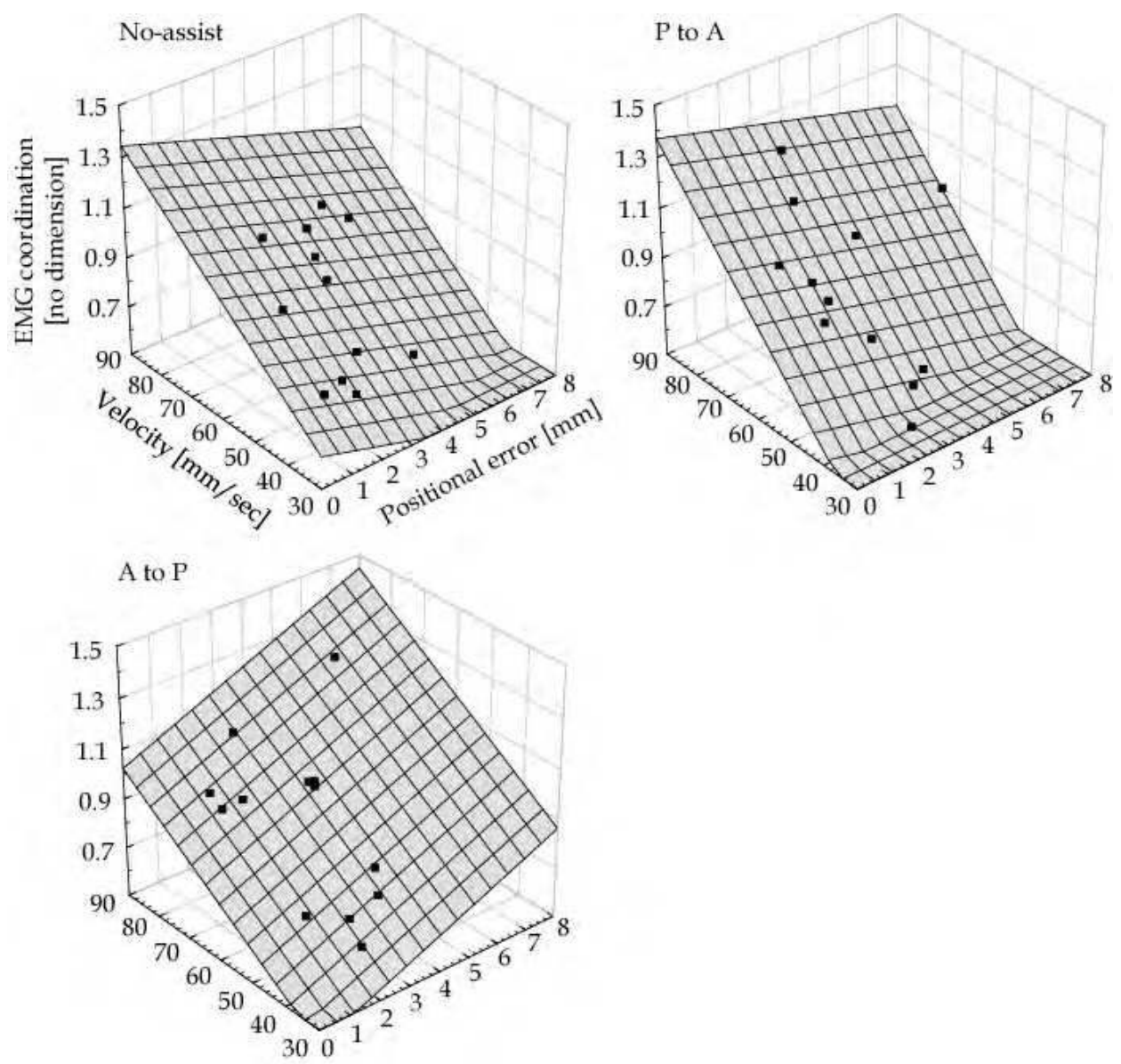

Fig. 12. Three-dimensional (3D) plots showing the relationship among the EMG coordination, positional error, and movement velocity in the same subject shown in Fig. 7 . The left top 3D plot shows the no-assist condition; the left bottom denotes the anterior-to-posterior condition; and the right top shows the posterior-to-anterior force applied condition. 
Another question is the intensity of the rehabilitation methods. This would depend on the remaining individual motor functions of each patient. In this regard, using the haptic device, the operators can easily change the magnitude and the direction of the virtual force. Namely, the haptic device could either induce the constraint movement using the large magnitudes of the virtual force or train against the resist force as the motor functions improve. In these respects, a robotic rehabilitation tool, specifically, the haptic device, would be an effective method for neurorehabilitation.
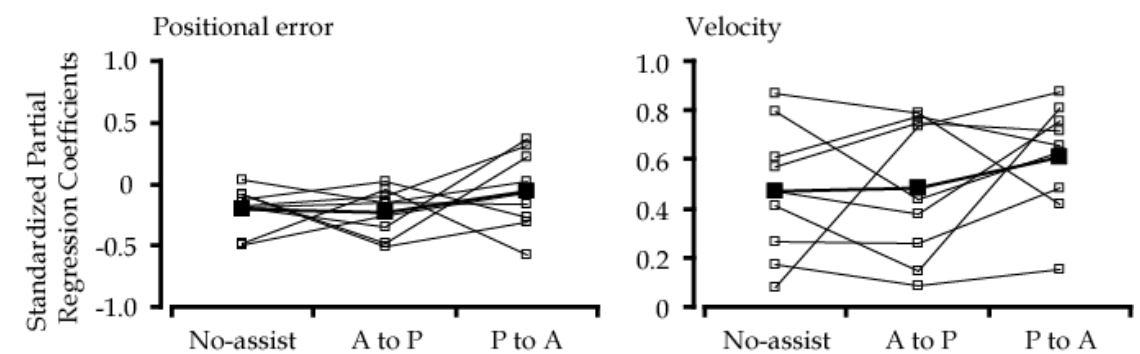

Fig. 13. Differences in sensitivity to both the positional error and the movement velocity. Although there are no significant differences in either of the standardized partial regression coefficients, the EMG coordination tends to increase with the posterior-to-anterior force applied in two-joint arm movements in a planar horizontal plane compared to those under the no-assist and the anterior-to-posterior force applied conditions. The open rectangles denote subject-to-subject changes, and the filled rectangles denote the grand averages.

In conclusion, we have developed the haptic device for upper limb motor rehabilitation, which can provide an assist/resist force on the grip. From the preliminary experimental results, the EMG data provided evidence of improved muscle activation patterns between the agonist and antagonist muscles by the application of the assist/resist force as well as of improved EMG coordination.

\section{References}

Aisen, M.L.; Krebs, H.I.; Hogan, N.; McDowell, F. \& Volpe, B.T. (1997). The effect of robot-assisted therapy and rehabilitative training on motor recovery following stroke. Archives of Neurology, Vol.54, No.4, 443-446, ISSN: 0003-9942

Colombo, G.; Joerg, M.; Schreier, R. \& Dietz, V. (2000). Treadmill training of paraplegic patients using a robotic orthosis. Journal of Rehabilitation Research and Development, Vol.37, No.6, 693-700, ISSN: 0748-7711

Dromerick, A.W.; Edwards, D.F. \& Hahn, M. (2000). Does the application of constraint-induced movement therapy during acute rehabilitation reduce arm impairment after ischemic stroke? Stroke, Vol.31, No.12, 2984-2988, ISSN: 0039-2499

Feys, H.M.; De Weerdt, W.J.; Selz, B.E.; Cox Steck, G.A.; Spichiger, R.; Vereeck, L.E.; Putman, K.D. \& Van Hoydonck, G.A. (1998). Effect of a therapeutic intervention for the hemiplegic upper limb in the acute phase after stroke: a single-blind, randomized, controlled multicenter trial. Stroke, Vol.29, No.4, 785-792, ISSN: 0039-2499

Fitts, P.M. (1954). The information capacity of the human motor system in controlling the amplitude of movements. Journal of Experimental Psychology, Vol.47, No.6, 381-391, ISSN:0022-1015 
Karst, G.M. \& Hasan, Z. (1991a). Initiation rules for planar, two-joint arm movements: agonist selection for movements throughout the work space. Journal of Neurophysiology, Vol.66, No.5, 1579-1593, ISSN: 0022-3077

Karst, G.M. \& Hasan, Z. (1991b). Timing and magnitude of electromyographic activity for two-joint arm movements in different directions. Journal of Neurophysiology, Vol.66, No.5, 1594-1604, ISSN: 0022-3077

Krebs, H.I.; Ferraro, M.; Buerger, S.P.; Newbery, M.J.; Makiyama, A.; Sandmann, M.; Lynch, D.; Volpe, B.T. \& Hogan, N. (2004). Rehabilitation robotics: pilot trial of a spatial extension for MIT-Manus. Journal of Neuroengineering and Rehabilitation, Vol.1, No.1, ISSN: 1743-0003

Lee, H.; Takahashi, Y.; Miyoshi, T.; Terada, T.; Inoue, K.; Ito, Y.; Ikeda, Y.; Suzuki, K. \& Komeda, T. (2005). Basic experiments of upper limb rehabilitation using haptic device system, Proceedings of the 2005 IEEE 9th International Conference on Rehabilitation Robotics, pp. 444-447, ISBN :0-7803-9003-2, Chicago, 28 June - 1 July 2005, IEEE

Lincoln, N.B.; Parry, R.H. \& Vass, C.D. (1999). Randomized, controlled trial to evaluate increased intensity of physiotherapy treatment of arm function after stroke. Stroke, Vol.30, No.3, 573-579, ISSN: 0039-2499

Lum, P.S.; Burgar, C.G.; Van der Loos, M.; Shor, P.C.; Majmundar, M. \& Yap, R. (2006). MIME robotic device for upper-limb neurorehabilitation in subacute stroke subjects: A follow-up study. Journal of Rehabilitation Research and Development, Vol.43, No.5, 631-642, ISSN: 0748-7711

Miltner, W.H.; Bauder, H.; Sommer, M.; Dettmers, C. \& Taub, E. (1999). Effects of constraint-induced movement therapy on patients with chronic motor deficits after stroke: a replication. Stroke, Vol.30, No.3, 586-592, ISSN: 0039-2499

Raasch, C.C., \& Zajac, F.E. (1999). Locomotor strategy for pedaling: muscle groups and biomechanical functions. Journal of Neurophysiology, Vol.82, No.2, 515-525, ISSN:0022-3077

Scheidt, R.A.; Reinkensmeyer, D.J.; Conditt, M.A.; Rymer, W.Z. \& Mussa-Ivaldi, F.A. (2000). Persistence of motor adaptation during constrained, multi-joint, arm movements. Journal of Neurophysiology, Vol.84, No.2, 853-862, ISSN: 0022-3077

Schwartz, A.B. \& Moran, D.W. (1999). Motor cortical activity during drawing movement: population representation during lemniscate tracing. Journal of Neurophysiology, Vol.82, No.5, 2705-2718, ISSN: 0022-3077

Sunderland, A.; Fletcher, D.; Bradley, L.; Tinson, D.; Hewer, R.L. \& Wade, D.T. (1994). Enhanced physical therapy for arm function after stroke: a one year follow up study. Journal of Neurology, Neurosurgery, and Psychiatry, Vol.57, No.7, 856-858, ISSN: 0022-3050

Takahashi, Y.; Terada, T.; Inoue, K.; Ito, Y.; Lee, H. \& Komeda, T. (2003). Upper-limb rehabilitation exercises using haptic device system. International Journal of Human-friendly Welfare Robotic Systems, Vol.4, No.2, 18-22, ISSN:1598-3250

Taub, E.; Miller, N.E.; Novack, T.A.; Cook, E.W.3rd; Fleming, W.C.; Nepomuceno, C.S.; Connell, J.S. \& Crago, J.E. Technique to improve chronic motor deficit after stroke. Archives of Physical Medicine and Rehabilitation, Vol.74, No.4, 347-354, ISSN: 0003-9993

Wernig, A. \& Muller, S. (1992). Laufband locomotion with body weight support improved walking in persons with severe spinal cord injuries. Paraplegia, Vol.30, No.4, 229-238, ISSN: 0031-1758 


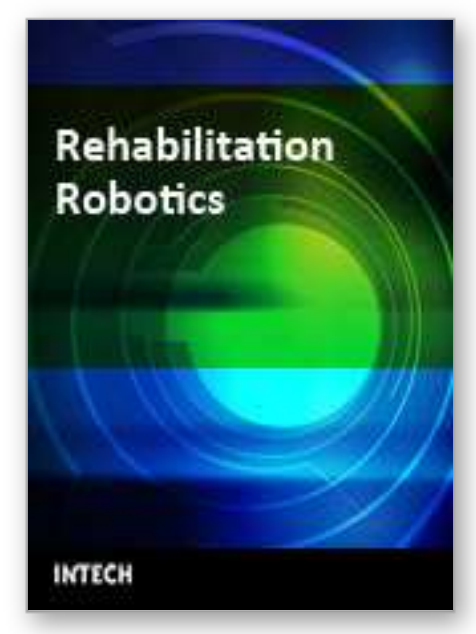

\author{
Rehabilitation Robotics \\ Edited by Sashi S Kommu
}

ISBN 978-3-902613-04-2

Hard cover, 648 pages

Publisher I-Tech Education and Publishing

Published online 01, August, 2007

Published in print edition August, 2007

The coupling of several areas of the medical field with recent advances in robotic systems has seen a paradigm shift in our approach to selected sectors of medical care, especially over the last decade.

Rehabilitation medicine is one such area. The development of advanced robotic systems has ushered with it an exponential number of trials and experiments aimed at optimising restoration of quality of life to those who are physically debilitated. Despite these developments, there remains a paucity in the presentation of these advances in the form of a comprehensive tool. This book was written to present the most recent advances in rehabilitation robotics known to date from the perspective of some of the leading experts in the field and presents an interesting array of developments put into 33 comprehensive chapters. The chapters are presented in a way that the reader will get a seamless impression of the current concepts of optimal modes of both experimental and ap- plicable roles of robotic devices.

\title{
How to reference
}

In order to correctly reference this scholarly work, feel free to copy and paste the following:

Tasuku Miyoshi, Yoshiyuki Takahashi, Hokyoo Lee, Takafumi Terada, Yuko Ito, Kaoru Inoue and Takashi Komeda (2007). Haptic Device System For Upper Limb Motor Impairment Patients: Developing And Handling In Healthy Subjects, Rehabilitation Robotics, Sashi S Kommu (Ed.), ISBN: 978-3-902613-04-2, InTech, Available from:

http://www.intechopen.com/books/rehabilitation_robotics/haptic_device_system_for_upper_limb_motor_impair ment_patients_developing_and_handling_in_healthy_su

\section{INTECH}

open science | open minds

\author{
InTech Europe \\ University Campus STeP Ri \\ Slavka Krautzeka 83/A \\ 51000 Rijeka, Croatia \\ Phone: +385 (51) 770447 \\ Fax: +385 (51) 686166 \\ www.intechopen.com
}

\author{
InTech China \\ Unit 405, Office Block, Hotel Equatorial Shanghai \\ No.65, Yan An Road (West), Shanghai, 200040, China \\ 中国上海市延安西路65号上海国际贵都大饭店办公楼 405 单元 \\ Phone: +86-21-62489820 \\ Fax: +86-21-62489821
}


(C) 2007 The Author(s). Licensee IntechOpen. This chapter is distributed under the terms of the Creative Commons Attribution-NonCommercial-ShareAlike-3.0 License, which permits use, distribution and reproduction for non-commercial purposes, provided the original is properly cited and derivative works building on this content are distributed under the same license. 